\title{
How to Review a Paper
}

\section{Including a Checklist for Hypothesis Testing Research Reports}

\author{
René Bekkers, Vrije Universiteit Amsterdam
}

June $24,2020^{*}$

Academia critically relies on our efforts as peer reviewers to evaluate the quality of research that is published in journals. Reading the reviews of others, I have noticed that the quality varies considerably, and that some reviews are not helpful. The added value of a journal article above and beyond the original manuscript or a non-reviewed preprint is in the changes the authors made in response to the reviews. Through our reviews, we can help to improve the quality of the research. This memo provides guidance on how to review a paper, partly inspired by suggestions provided by Alexander (2005), Lee (1995) and the Committee on Publication Ethics (2017). To improve the quality of the peer review process, I suggest that you use the following guidelines. Some of the guidelines - particularly the criteria at the end of this post - are peculiar for the kind of research that I tend to review - hypothesis testing research reports relying on administrative data and surveys, sometimes with an experimental design. But let me start with guidelines that I believe make sense for all research.

\section{Things to check before you accept the invitation}

First, I encourage you to check whether the journal aligns with your vision of science. I find that a journal published by an exploitative publisher making a profit in the range of $30 \%-40 \%$ is not worth my time. A journal that I have submitted my own work to and gave me good reviews is worth the number of reviews I received for my article. The review of a revised version of the paper does not count as a separate paper.

Next, I check whether I am the right person to review the paper. I think it is a good principle to describe my disciplinary background and expertise in relation to the manuscript I am invited to review. Reviewers do not need to be experts in all respects. If you do not have useful expertise to improve the paper, politely decline.

Then I check whether I know the author(s). If I do, and I have not collaborated with the author(s), if I am not currently collaborating or planning to do so, I describe how I know the author(s) and ask the editor whether it is appropriate for me to review the paper. If I have a

\footnotetext{
* This work is licensed under a Creative Commons Attribution 4.0 International License. Thanks to colleagues at the Center for Philanthropic Studies at Vrije Universiteit Amsterdam, in particular Pamala Wiepking, Arjen de Wit, Theo Schuyt and Claire van Teunenbroek, for insightful comments on the first version. Thanks to Robin Banks, Pat Danahey Janin, Rense Corten, David Reinstein, Eleanor Brilliant, Claire Routley, Margaret Harris, Brenda Bushouse, Craig Furneaux, Angela Eikenberry, Jennifer Dodge, and Tracey Coule for responses to the second draft. The current text is the fourth draft. The most recent version of this paper is available as a preprint at https://doi.org/10.31219/osf.io/7ug4w. Suggestions continue to be welcome at r.bekkers@vu.nl
} 
conflict of interest, I notify the editor and politely decline. It is a good principle to let the editor know immediately if you are unable to review a paper, so the editor can start to look for someone else to review the paper. Your non-response means a delay for the authors and the editor.

Sometimes I get requests to review a paper that I have reviewed before, for a conference or another journal. In these cases I let the editor know and ask the editor whether she would like to see the previous review. For the editor it will be useful to know whether the current manuscript is the same as the version, or includes revisions.

Finally, I check whether the authors have made the data and code available. I have made it a requirement that authors have to fulfil before I accept an invitation to review their work. An exception can be made for data that would be illegal or dangerous to make available, such as datasets that contain identifying information that cannot be removed. In most cases, however, the authors can provide at least partial access to the data by excluding variables that contain personal information.

A paper that does not provide access to the data analyzed and the code used to produce the results in the paper is not worth my time. If the paper does not provide a link to the data and the analysis script, I ask the editor to ask the authors to provide the data and the code. I encourage you to do the same. Almost always the editor is willing to ask the authors to provide access. If the editor does not respond to your request, that is a red flag to me. I decline future invitation requests from the journal. If the authors do not respond to the editor's request, or are unwilling to provide access to the data and code, that is a red flag for the editor.

\section{The tone of the review}

When I write a review, I think of the 'golden rule': treat others as you would like to be treated. I write the review report that I would have liked to receive if I had been the author. I use the following principles:

- Be honest but constructive. You are not at war. There is no need to burn a paper to the ground.

- Avoid addressing the authors personally. Say: "the paper could benefit from..." instead of "the authors need".

- Stay close to the facts. Do not speculate about reasons why the authors have made certain choices beyond the arguments stated in the paper.

- Take a developmental approach. Any paper will contain flaws and imperfections. Your job is to improve science by identifying problems and suggesting ways to repair them. Think with the authors about ways they can improve the paper in such a way that it benefits collective scholarship. After a quick glance at the paper, I determine whether I think the paper has the potential to be published, perhaps after revisions. If I think the paper is beyond repair, I explain this to the editor.

- Try to see beyond bad writing style and mistakes in spelling. Also be mindful of disciplinary and cultural differences between the authors and yourself. 


\section{The substance of the advice}

In my view, it is a good principle to begin the review report by describing your expertise and the way you reviewed the paper. If you searched for literature, checked the data and verified the results, ran additional analyses, state this. It will allow the editor to adjudicate the review.

Then give a brief overview of the paper. If the invitation asks you to provide a general recommendation, consider whether you'd like to give one. Typically, you are invited to recommend 'reject', 'revise $\&$ resubmit' - with major or minor revisions, or 'accept'. Because the recommendation is the first thing the editor wants to know it is convenient to state it early in the review.

When giving such a recommendation, I start from the assumption that the authors have invested a great deal of time in the paper and that they want to improve it. Also I consider the desk-rejection rate at the journal. If the editor sent the paper out for review, she probably thinks it has the potential to be published.

To get to the general recommendation, I list the strengths and the weaknesses of the paper. To ease the message you can use the sandwich principle: start with the strengths, then discuss the weaknesses, and conclude with an encouragement.

For authors and editors alike it is convenient to give actionable advice. For the weaknesses in the paper I suggest ways to repair them. I distinguish major issues such as not discussing alternative explanations from minor issues such as missing references and typos. It is convenient for both the editor and the authors to number your suggestions.

The strengths could be points that the authors are underselling. In that case, I identify them as strengths that the authors can emphasize more strongly.

It is handy to refer to issues with direct quotes and page numbers. To refer to the previous sentence: "As the paper states on page 3, [use] "direct quotes and page numbers"”.

In 2016 I have started to sign my reviews. This is an accountability device: by exposing who I am to the authors of the paper I'm reviewing, I set higher standards for myself. I encourage you to think about this as an option, though I can imagine that you may not want to risk retribution as a graduate student or an early career researcher. Also some editors do not appreciate signed reviews and may take away your identifying information.

\section{How to organize the review work}

Usually, I read a paper twice. First, I go over the paper superficially and quickly. I do not read it closely. This gets me a sense of where the authors are going. After the first superficial reading, I determine whether the paper is good enough to be revised and resubmitted, and if so, I provide more detailed comments. After the report is done, I revisit my initial recommendation.

The second time I go over the paper, I do a very close reading. Because the authors had a word limit, I assume that literally every word in the manuscript is absolutely necessary - the paper 
should have no repetitions. Some of the information may be in the supplementary information provided with the paper.

Below you find a checklist of things I look for in a paper. The checklist reflects the kind of research that I tend to review, which is typically testing a set of hypotheses based on theory and previous research with data from surveys, experiments, or archival sources. For other types of research - such as non-empirical papers, exploratory reports, and studies based on interviews or ethnographic material - the checklist is less appropriate. The checklist may also be helpful for authors preparing research reports.

I realize that this is an extensive set of criteria for reviews. It sets the bar pretty high. A review checking each of the criteria will take you at least three hours, but more likely between five and eight hours. As a reviewer, I do not always check all criteria myself. Some of the criteria do not necessarily have to be done by peer reviewers. For instance, some journals employ data editors who check whether data and code provided by authors produce the results reported.

I do hope that journals and editors can get to a consensus on a set of minimum criteria that the peer review process should cover, or at least provide clarity about the criteria that they do check.

\section{Review checklist for hypothesis testing reports}

\section{$\underline{\text { Research question }}$}

1. Is it clear from the beginning what the research question is? If it is in the title, that's good. In the first part of the abstract is good too. Is it at the end of the introduction section? In most cases that is too late.

2. Is it clearly formulated? By the research question alone, can you tell what the paper is about?

3. Does the research question align with what the paper actually does - or can do - to answer it?

4. Is it important to know the answer to the research question for previous theory and methods?

5. Does the paper address a question that is important from a societal or practical point of view?

\section{$\underline{\text { Research design }}$}

1. Does the research design align with the research question? If the question is descriptive, do the data actually allow for a representative and valid description? If the question is a causal question, do the data allow for causal inference? If not, ask the authors to report 'associations' rather than 'effects'.

2. Is the research design clearly described? Does the paper report all the steps taken to collect the data? 
3. Does the paper identify mediators of the alleged effect? Does the paper identify moderators as boundary conditions?

4. Is the research design waterproof? Does the study allow for alternative interpretations?

5. Has the research design been preregistered? Does the paper refer to a public URL where the preregistration is posted? Does the preregistration include a statistical power analysis? Is the number of observations sufficient for statistical tests of hypotheses? Are deviations from the preregistered design reported?

6. Has the experiment been approved by an Internal or Ethics Review Board (IRB/ERB)? What is the IRB registration number?

Theory

1. Does the paper identify multiple relevant theories?

2. Does the theory section specify hypotheses? Have the hypotheses been formulated before the data were collected? Before the data were analyzed?

3. Do hypotheses specify arguments why two variables are associated? Have alternative arguments been considered?

4. Is the literature review complete? Does the paper cover the most relevant previous studies, also outside the discipline? Provide references to research that is not covered in the paper, but should definitely be cited.

\section{$\underline{\text { Data \& Methods }}$}

1. Target group - Is it identified? If mankind, is the sample a good sample of mankind? Does it cover all relevant units?

2. Sample - Does the paper identify the procedure used to obtain the sample from the target group? Is the sample a random sample? If not, has selective non-response been dealt with, examined, and have constraints on generality been identified as a limitation?

3. Number of observations - What is the statistical power of the analysis? Does the paper report a power analysis?

4. Measures - Does the paper provide the complete topic list, questionnaire, instructions for participants? To what extent are the measures used valid? Reliable?

5. Descriptive statistics - Does the paper provide a table of descriptive statistics (minimum, maximum, mean, standard deviation, number of observations) for all variables in the analyses? If not, ask for such a table.

6. Outliers - Does the paper identify treatment of outliers, if any?

7. Is the multi-level structure (e.g., persons in time and space) identified and taken into account in an appropriate manner in the analysis? Are standard errors clustered?

8. Does the paper report statistical mediation analyses for all hypothesized explanation(s)? Do the mediation analyses evaluate multiple pathways, or just one?

9. Do the data allow for testing additional explanations that are not reported in the paper? 
$\underline{\text { Results }}$

1. Can the results be reproduced from the data and code provided by the authors?

2. Are the results robust to different specifications?

\section{Conclusion}

1. Does the paper give a clear answer to the research question posed in the introduction?

2. Does the paper identify implications for the theories tested, and are they justified?

3. Does the paper identify implications for practice, and are they justified given the evidence presented?

\section{$\underline{\text { Discussion }}$}

1. Does the paper revisit the limitations of the data and methods?

2. Does the paper suggest future research to repair the limitations?

$\underline{\text { Meta }}$

1. Does the paper have an author contribution note? Is it clear who did what?

2. Are all analyses reported, if they are not in the main text, are they available in an online appendix?

3. Are references up to date? Does the reference list include a reference to the dataset analyzed, including an URL/DOI?

\section{After the review}

If the authors have revised their paper, it is a good principle to avoid making new demands for the second round that you have not made before. Otherwise the revise and resubmit path can be very long.

\section{References}

Alexander, G.R. (2005). A Guide to Reviewing Manuscripts. Maternal and Child Health Journal, 9 (1): 113-117. https://doi.org/10.1007/s10995-005-2423-y

Committee on Publication Ethics Council (2017). Ethical guidelines for peer reviewers. https://publicationethics.org/files/Ethical_Guidelines_For_Peer_Reviewers_2.pdf

Lee, A.S. (1995). Reviewing a manuscript for publication. Journal of Operations Management, 13: 87-92. https://doi.org/10.1016/0272-6963(95)94762-W 